\title{
SIEGEL DISKS AND RENORMALIZATION FIXED POINTS
}

\author{
MICHAEL YAMPOLSKY
}

\begin{abstract}
In this note we construct hyperbolic fixed points for cylinder renormalization of maps with Siegel disks.
\end{abstract}

\section{INTRODUCTION}

A renormalization hyperbolicity conjecture has so far been established in two examples of one-dimensional dynamical systems: the unimodal maps, in the works of Sullivan Sul1, Sul2, MvS, McMullen McM1, McM2, and Lyubich Lyu4, Lyu5; and the critical circle maps, in the works of de Faria and de Melo dF1, dF2, dFdM1, dFdM2, and the author [Ya2, Ya3, Ya4]. In this paper we will add one more example to the list, by constructing a hyperbolic fixed points of renormalization corresponding to Siegel disks. Let us say that an irrational number $\theta$ is golden if it is represented by an infinite continued fraction

$$
\theta=\frac{1}{N+\frac{1}{N+\frac{1}{\cdots}}} \text {, with } N \in \mathbb{N} .
$$

We introduce this notation by analogy with the golden mean $(\sqrt{5}-1) / 2$ which is expressed by such a fraction with $N=1$. It has long been known, that golden Siegel disks in the quadratic family have self-similar scaling properties near the critical point, explained by a renormalization hyperbolicity conjecture [MN, Wi]. MacKay and Persival [MP] have conjectured in 1986, based on numerical evidence, the existence of a hyperbolic renormalization horseshoe corresponding to Siegel disks of analytic maps, analogous to the Lanford's horseshoe for critical circle maps [Lan1, Lan2].

In 1994 Stirnemann [Stir] gave a computer-assisted proof of the existence of a renormalization fixed point with a golden-mean Siegel disk. In 1998, McMullen [McM3] proved the asymptotic self-similarity of golden Sigel disks in the quadratic family. He constructed a version of renormalization based on holomorphic commuting pairs of de Faria dF1, dF2] and showed that the renormalizations of a quadratic polynomial with a golden Siegel disk near the critical point converge to a fixed point geometrically fast. More generally, he constructed a renormalization horseshoe for bounded type rotation numbers, and used

Date: April 10, 2018.

This note was written in the Fall of 2003, and amended with a corollary of the new results of M. Shishikura in the Summer of 2005. 
renormalization to show that the Hausdorff dimension of the corresponding quadratic Julia sets is strictly less than two.

In Ya3 we introduced a new renormalization transformation $\mathcal{R}_{\text {cyl }}$, which we called the cylinder renormalization, and used it to prove the Lanford's Hyperbolicity Conjecture for critical circle maps. The main advantage of $\mathcal{R}_{\text {cyl }}$ over the renormalization scheme based on commuting pairs is that this operator is analytic in a Banach manifold of analytic maps of a subdomain of $\mathbb{C} / \mathbb{Z}$. In this paper we use McMullen's result to construct a fixed point $\hat{f}$ of $\mathcal{R}_{\text {cyl }}$ with a golden-mean Siegel disk. We further discuss the properties of $\mathcal{R}_{\text {cyl }}$ at the fixed point $\hat{f}$, and, in particular, show that its linearization is a compact operator having at least one eigenvalue outside the closed unit disk.

Finally, we use the new results of Inou and Shishikura [Shi2] to show that for sufficiently large values of $N$, this fixed point of $\mathcal{R}_{\mathrm{c} y l}$ is hyperbolic, and the dimension of its expanding subspace is exactly one.

Theorem 1.1. Let

$$
\theta_{N}=\frac{1}{N+\frac{1}{N+\frac{1}{\cdots}}} \text { with } N \in \mathbb{N}
$$

be a golden number. There exists a complex Banach space $\mathbf{C}_{U(N)}$ whose elements (referred to as critical cylinder maps) are analytic maps defined in a neighborhood $U(N)$ of the origin, such that the following holds. There exists a critical cylinder map $\hat{f}_{N} \in \mathbf{C}_{U(N)}$ with a Siegel disk $\Delta \Subset U(N)$ with rotation number $\theta_{N}$ for which:

(I) the boundary of $\Delta$ is a quasicircle containing the critical point of $\hat{f}_{N}$;

(II) $\mathcal{R}_{c y l} \hat{f}_{N}=\hat{f}_{N}$;

(III) the quadratic polynomial $f(z)=e^{2 \pi i \theta_{N}} z+z^{2}$ is infinitely cylinder renormalizable, and

$$
\mathcal{R}_{c y l}^{k} f \rightarrow \hat{f}_{N}
$$

at a uniform geometric rate;

(IV) the cylinder renormalization $\mathcal{R}_{c y l}$ is an analytic and compact operator mapping a neighborhood of the fixed point $\hat{f}_{N}$ in $\mathbf{C}_{U(N)}$ to $\mathbf{C}_{U(N)}$. Its linearization at $\hat{f}_{N}$ is a compact operator, with at least one eigenvalue with the absolute value greater than one;

$(\mathrm{V})$ there exists a neighborhood $U_{\infty}$ of the origin which is contained in all $U(N)$ for large enough $N$.

Moreover, there exists $N_{0} \in \mathbb{N}$ such that for all $N \geq N_{0}$ we have:

(VI) except for the one unstable eigenvalue, the spectrum of $\mathcal{R}_{c y l}$ at $\hat{f}_{N}$ is compactly contained inside the unit disk.

Remark 1.1. We note that the results (I)-(V) extend to show the existence of an invariant horseshoe for renormalization of Siegel disks with rotation numbers $\theta$ of type bounded by 
any $B \in \mathbb{N}$. The result (VI) extends to the horseshoe of Siegel disks with bounded type for which every term in the continued fraction expansion of $\theta_{0}$ is at least $N_{0}$. We restrict ourselves to the case of a renormalization fixed point for simplicity of exposition.

Acknowledgements. I would like to thank Xavier Buff for several useful discussions of the results of Inou and Shishikura, and for suggesting a different argument for proving Theorem 2.12. In addition, I would like to thank X. Buff and A. Chéritat for discussing the Doaudy's Program of constructing positive measure Julia sets with me.

\section{Preliminary CONSIDERATions.}

Some notations. We use dist and diam to denote the Euclidean distance and diameter in $\mathbb{C}$. We shall say that two real numbers $A$ and $B$ are $K$-commensurable for $K>1$ if $K^{-1}|A| \leq|B| \leq K|A|$. The notation $D_{r}(z)$ will stand for the Euclidean disk with the center at $z \in \mathbb{C}$ and radius $r$. The unit disk $D_{1}(0)$ will be denoted $\mathbb{D}$. The plane $(\mathbb{C} \backslash \mathbb{R}) \cup J$ with the parts of the real axis not contained in the interval $J \subset \mathbb{R}$ removed will be denoted $\mathbb{C}_{J}$. By the circle $\mathbb{T}$ we understand the affine manifold $\mathbb{R} / \mathbb{Z}$, it is naturally identified with the unit circle $S^{1}=\partial \mathbb{D}$. The real translation $x \mapsto x+\theta$ projects to the rigid rotation by angle $\theta, R_{\theta}: \mathbb{T} \rightarrow \mathbb{T}$, the same map on $S^{1}$ will be denoted $r_{\theta}$. For two points $a$ and $b$ in the circle $\mathbb{T}$ which are not diametrically opposite, $[a, b]$ will denote the shorter of the two arcs connecting them. As usual, $|[a, b]|$ will denote the length of the arc. For two points $a, b \in \mathbb{R},[a, b]$ will denote the closed interval with endpoints $a, b$ without specifying their order. The cylinder in this paper, unless otherwise specified will mean the affine manifold $\mathbb{C} / \mathbb{Z}$. Its equator is the circle $\{\operatorname{Im} z=0\} / \mathbb{Z} \subset \mathbb{C} / \mathbb{Z}$. A topological annulus $A \subset \mathbb{C} / \mathbb{Z}$ will be called an equatorial annulus, or an equatorial neighborhood, if it has a smooth boundary and contains the equator.

By "smooth" in this paper we will mean "of class $C^{\infty}$ ", unless another degree of smoothness is specified. The notation " $C \omega$ " will stand for "real-analytic".

We will sometimes use a symbol $\infty$ alongside the natural numbers, with the usual conventions $\infty>n, 1 / \infty=0$, and $\infty \pm n=\infty$ for $n \in \mathbb{N}$.

Renormalization of critical circle maps. We are going to recall here very briefly the way renormalization of critical circle maps is defined using commuting pairs [FKS, ORSS]. A detailed account of what follows may be found in Ya3. A critical circle mapping $f$ is a homeomorphism $\mathbb{T} \rightarrow \mathbb{T}$ of class $C^{3}$ with an only critical point at 0 . The latter is further assumed to be non-flat, usually cubic. Yoccoz [Yoc] has shown that if such a mapping has an irrational rotation number $\rho(f)$, then it is conjugate to the rigid rotation of the circle by angle $\rho(f)$ by a homeomorphic change of coordinate. Writing $\rho(f)$ as an infinite continued fraction with positive terms

$$
\rho(f)=\frac{1}{a_{1}+\frac{1}{a_{2}+\frac{1}{\ldots}}}
$$


(which we will further abbreviate as $\left[a_{1}, a_{2}, \ldots\right]$ ), we denote $\left\{p_{n} / q_{n}\right\}$ the sequence of its convergents

$$
p_{n} / q_{n}=\left[a_{1}, \ldots, a_{n}\right]
$$

As $p_{n} / q_{n}$ are best rational approximations of $\rho(f)$, the denominators $q_{n}$ are closest return times of the critical point 0 : the arc $J_{n}=\left[0, f^{q_{n}}(0)\right]$ contains no iterates $f^{j}(0)$ with $j<q_{n}$. The first return map of $J_{n} \cup J_{n+1}$ is a piecewise defined mapping given by

$$
R_{J_{n} \cup J_{n+1}} f=\left(\left.f^{q_{n+1}}\right|_{J_{n}},\left.f^{q_{n}}\right|_{J_{n+1}}\right) .
$$

This serves as a motivation for the following definition.

Definition 2.1. A commuting pair $\zeta=(\eta, \xi)$ consists of two $C^{3}$-smooth orientation preserving interval homeomorphisms $\eta: I_{\eta} \rightarrow \eta\left(I_{\eta}\right), \xi: I_{\xi} \rightarrow \xi\left(I_{\xi}\right)$, where

(I) $I_{\eta}=[0, \xi(0)], I_{\xi}=[\eta(0), 0]$;

(II) Both $\eta$ and $\xi$ have homeomorphic extensions to interval neighborhoods of their respective domains with the same degree of smoothness, which commute, $\eta \circ \xi=\xi \circ \eta$;

(III) $\xi \circ \eta(0) \in I_{\eta}$;

(IV) $\eta^{\prime}(x) \neq 0 \neq \xi^{\prime}(y)$, for all $x \in I_{\eta} \backslash\{0\}$, and all $y \in I_{\xi} \backslash\{0\}$.

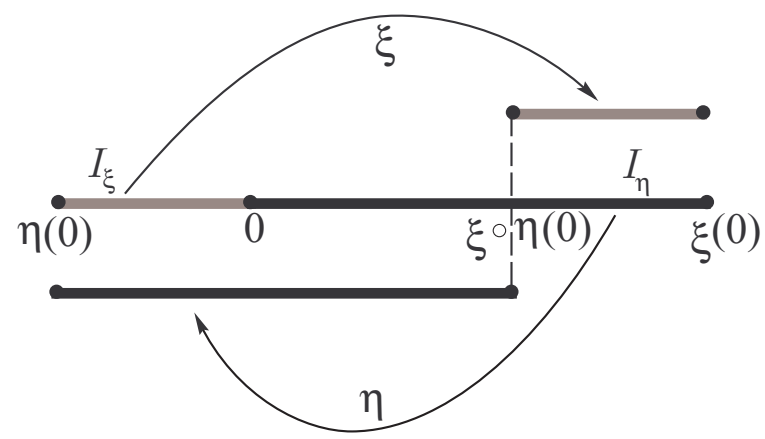

Figure 1. A commuting pair

The height $\chi(\zeta)$ of a critical commuting pair $\zeta=(\eta, \xi)$ is equal to $r$, if

$$
0 \in\left[\eta^{r}(\xi(0)), \eta^{r+1}(\xi(0))\right] .
$$

If no such $r$ exists, we set $\chi(\zeta)=\infty$, in this case the map $\eta \mid I_{\eta}$ has a fixed point. For a pair $\zeta$ with $\chi(\zeta)=r<\infty$ one verifies directly that the mappings $\eta \mid\left[0, \eta^{r}(\xi(0))\right]$ and $\eta^{r} \circ \xi \mid I_{\xi}$ again form a commuting pair. For a commuting pair $\zeta=(\eta, \xi)$ we will denote by $\widetilde{\zeta}$ the pair $\left(\widetilde{\eta}\left|\widetilde{I}_{\eta}, \widetilde{\xi}\right| \widetilde{I}_{\xi}\right)$ where tilde means rescaling by the linear factor $\lambda=-\frac{1}{\left|I_{\eta}\right|}$.

Definition 2.2. The renormalization of a real commuting pair $\zeta=(\eta, \xi)$ is the commuting pair 


$$
\mathcal{R} \zeta=\left(\widetilde{\eta^{r} \circ \xi}\left|\widetilde{I}_{\xi}, \widetilde{\eta}\right|\left[0, \widetilde{\eta^{r}(\xi(0))}\right]\right) .
$$

For a pair $\zeta$ we define its rotation number $\rho(\zeta) \in[0,1]$ to be equal to the continued fraction $\left[r_{0}, r_{1}, \ldots\right]$ where $r_{i}=\chi\left(\mathcal{R}^{i} \zeta\right)$. In this definition $1 / \infty$ is understood as 0 , hence a rotation number is rational if and only if only finitely many renormalizations of $\zeta$ are defined; if $\chi(\zeta)=\infty, \rho(\zeta)=0$.

The non-rescaled pair $\left(\eta^{r} \circ \xi\left|I_{\xi}, \eta\right|\left[0, \eta^{r}(\xi(0))\right]\right)$ will be referred to as the pre-renormalization $p \mathcal{R} \zeta$ of the commuting pair $\zeta=(\eta, \xi)$. For a critical circle map $f$ as above, we set

$$
p \mathcal{R}^{n} f=R_{J_{n} \cup J_{n+1}} f=\left(\left.f^{q_{n+1}}\right|_{J_{n}},\left.f^{q_{n}}\right|_{J_{n+1}}\right) \text {, and } \mathcal{R}^{n} f=\widetilde{p \mathcal{R}^{n} f} .
$$

A key object in the renormalization theory of commuting pairs developed by de Faria dF1, $\mathrm{dF} 2$ is the holomorphic commuting pair. This is an analogue of the Douady-Hubbard's polynomial-like map in the unimodal renormalization theory, which is defined as follows (cf. Figure 2):

Definition 2.3. An analytic commuting pair $\zeta=\left(\left.\eta\right|_{I_{\eta}},\left.\xi\right|_{I_{\xi}}\right)$ extends to a holomorphic commuting pair $\mathcal{H}$ if there exist four simply-connected $\mathbb{R}$-symmetric domains $V, U_{1}, U_{2}$, $U_{3}$ such that

- $\bar{U}_{1}, \bar{U}_{2}, \bar{U}_{3} \subset V, \bar{U}_{1} \cap \bar{U}_{2}=\{0\}$; the sets $U_{1} \backslash U_{3}, U_{2} \backslash U_{3}, U_{3} \backslash U_{1}$, and $U_{3} \backslash U_{2}$ are nonempty, connected, and simply-connected, $U_{1} \cap \mathbb{R}=I_{\eta}, U_{2} \cap \mathbb{R}=I_{\xi}$;

- mappings $\eta: U_{1} \rightarrow(V \backslash \mathbb{R}) \cup \eta\left(I_{\eta}\right)$ and $\xi: U_{2} \rightarrow(V \backslash \mathbb{R}) \cup \xi\left(I_{\xi}\right)$ are onto and univalent;

- $\nu \equiv \eta \circ \xi: U_{3} \rightarrow(V \backslash \mathbb{R}) \cup \nu\left(I_{U_{3}}\right)$ is a three-fold branched covering with a unique critical point at zero, where $I_{U_{3}}=U_{3} \cap \mathbb{R}$.

One says that an analytic commuting pair $(\eta, \xi)$ with an irrational rotation number has complex a priori bounds, if all its renormalizations extend to holomorphic commuting pairs with bounded moduli:

$$
\bmod \left(V \backslash \overline{\cup U_{i}}\right)>\mu>0
$$

Part of the significance of the complex a priori bounds is explained by the following:

Proposition 2.1 (Ya1]). For $\mu \in(0,1)$ let $\mathbf{H}(\mu)$ denote the space of holomorphic commuting pairs $\mathcal{H}$, with $\bmod \left(V \backslash \overline{\cup U_{i}}\right)>\mu, \min \left(\left|I_{\eta}\right|,\left|I_{\xi}\right|\right)>\mu$ and $\operatorname{diam}(V)<1 / \mu$. Then the space $\mathbf{H}(\mu)$ is sequentially pre-compact with respect to the Carathéodory topology, with all the limit points contained in $\mathbf{H}(\mu / 2)$.

The existense of complex a priori bounds is a key analytic issue of renormalization theory. In the case of critical circle maps it is settled by the following theorem:

Theorem 2.2. There exist universal constants $\mu>0$ and $K>1$ such that the following holds. Let $\zeta$ be an analytic critical commuting pair with an irrational rotation number. Then there exists $N=N(\zeta)$ such that for all $n \geq N$ the commuting pair $\mathcal{R}^{n} \zeta$ extends to a holomorphic commuting pair $\mathcal{H}_{n} \in \mathbf{H}(\mu)$. Moreover, its range $V_{n}$ is a Euclidean disk, and the regions $U_{i} \cap( \pm \mathbb{H})$ are $K$-quasidisks. 
We first proved this theorem in Ya1 for commuting pairs $\zeta$ in the Epstein class. Our proof was later adapted by de Faria and de Melo dFdM2 to the case of a non-Epstein analytic commuting pair.

Finally, let us make the following note for future reference.

Proposition 2.3. If $f$ be a homeomorphism of a topological circle $S$ with $\rho(f) \in \mathbb{R} \backslash \mathbb{Q}$. Fix a point $p \in S$ and let $A_{n}$ be the arc of the circle $S$ connecting $p$ to $f^{q_{n}}(p)$ and such that $f^{q_{n-1}}(p) \notin A_{n}$. Then for every $n \in \mathbb{N}$ the topological arcs

$$
A_{n}, f\left(A_{n}\right), \ldots, f^{q_{n+1}-1}\left(A_{n}\right), A_{n+1}, f\left(A_{n+1}\right), \ldots, f^{q_{n}-1}\left(A_{n+1}\right)
$$

cover the whole circle and have disjoint interiors.

We will call the intervals (2.1) the $n$-th dynamical partition of $f$, given by the orbit of $p$.

Siegel quadratics. Let $f(z)$ be a germ of an anlytic mapping at 0 , for which the origin is a fixed point. We will concentrate on the case when 0 is an irrationally indifferent fixed point of $f$, that is, $f^{\prime}(0)=e^{2 \pi i \theta}$, with the rotation number $\theta \in \mathbb{R} \backslash \mathbb{Q}$. The germ $f$ is linearizable, if after a conformal change of coordinate in a neighborhood of the origin, it becomes a rotation. The first such linearization result is due to Siegel [Sieg, we recall it below:

Theorem 2.4 (Sieg ). Suppose $\theta \in(0,1)$ is an irrational number of bounded type ${ }^{1}$ that is, it is represented by an infinite continued fraction with positive terms $\theta=\left[a_{1}, a_{2}, \ldots, a_{n}, \ldots\right]$ such that $\sup a_{i}<\infty$. Then any analytic germ $f(z)$ with the rotation number $\theta$ is linearizable.

For an analytic mapping $f$ with a linearizable irrational indifferent fixed point, the maximal linearization domain is called a Siegel disk. In the cases when $f$ has a natural domain of definition, the structure of the boundary of the Siegel disk allows further study. Let us consider the particular example of a quadratic polynomial

$$
f_{\theta}(z)=e^{2 \pi i \theta} z+z^{2}
$$

with $\theta$ an irrational of bounded type. The following properties then hold true:

Theorem 2.5 (Siegel quadratics of bounded type). Denote $\Delta$ the Siegel disk of $f_{\theta}$.

(I) The boundary of the Siegel disk $\Delta$ is a quasicircle containing the critical point of $f_{\theta}$.

(II) The Julia set of $f_{\theta}$ is locally connected.

(III) The Julia set of $f_{\theta}$ has zero area.

The second and third statements are theorems of Petersen [Pet] (see also [Ya1] for a different proof). The first statement is derived from the real a priori bounds for critical circle maps of Światek [Sw] and Herman [ $[\mathrm{Her}$, via a quasiconformal surgery procedure due to Douady,

\footnotetext{
1 "Bounded type" is the terminology of the renormalization theory, the term commonly used by the number theorists is "constant type".
} 
Ghys, Herman, and Shishikura. We will make use of this surgery further in the paper, let us therefore briefly recall it below.

Proof of (I). Consider the one-parameter family of degree three Blaschke products

$$
Q_{\tau}(z)=e^{2 \pi i \tau} z^{2} \frac{z-3}{1-3 z}
$$

For each value of $\tau$ the restriction $\left.Q_{\tau}\right|_{S^{1}}$ is an analytic critical circle mapping, with critical point $c=1$ and critical value $Q_{\tau}(1)=e^{2 \pi i \tau}$. For each irrational number $\theta \in \mathbb{T} \backslash \mathbb{Q}$ let us denote $\tau(\theta) \in \mathbb{T}$ the unique value of the parameter for which the rotation number of $\left.Q_{\tau(\theta)}\right|_{S^{1}}$ is equal to $\theta$ and set

$$
F_{\theta} \equiv Q_{\tau(\theta)}
$$

Consider the rigid rotation $r_{\theta}(z)=e^{2 \pi i \theta} z$ and let $\phi$ be the conjugacy

$$
\left.\phi \circ F_{\theta}\right|_{S^{1}} \circ \phi=\left.r_{\theta}\right|_{S^{1}}
$$

for which $\phi(1)=1$. By $[\mathrm{Sw}$ and $[\mathrm{Her}]$ if $\theta$ is of bounded type, then $\phi$ is a quasisymmetric map. Let us select a quasiconformal extension of $\phi$ to the unit disk $\mathbb{D}$. A new dynamical system $\tilde{F}_{\theta}$ will be defined as

$$
\tilde{F}_{\theta}=\left\{\begin{array}{l}
F_{\theta}(z), \text { when } z \notin \mathbb{D}, \\
\phi^{-1} \circ r_{\theta} \circ \phi(z), \text { when } z \in \mathbb{D}
\end{array}\right.
$$

We define a new complex structure $\mu$ in $\mathbb{D}$ setting it equal to the pull-back of the standard complex structure $\phi^{*} \sigma_{0}$. We extend $\mu$ to the outside of $\mathbb{D}$ setting it equal to $\left(\tilde{F}_{\theta}^{n}\right)^{*} \circ \phi^{*} \sigma_{0}$ for a point $z$ such that $\tilde{F}_{\theta}^{n}(z) \in \mathbb{D}$ and equal to $\sigma_{0}$ elsewhere. By construction, $\tilde{F}_{\theta}^{*} \mu=\mu$. By the Measurable Riemann Mapping Theorem, there exists a quasiconformal mapping $\psi: \hat{C} \rightarrow \hat{C}$ such that

$$
\psi^{*} \sigma_{0}=\mu \text { a.e., and } \psi(\infty)=\infty, \psi(0)=0, \psi(1)=-e^{2 \pi i \theta} / 2
$$

Then

$$
f_{\theta}=\psi^{-1} \circ \tilde{F}_{\theta} \circ \psi
$$

and the proof is complete.

McMullen's results on renormalization of Siegel disks of bounded type. For the polynomial $f_{\theta}$ as in Theorem 2.5 the restriction to $\partial \Delta$ is topologically conjugate to $R_{\theta}$. If $c \in \partial \Delta$ is the critical point of $f_{\theta}$, it is natural again (cf. [MN, Wi, MP]) to define the $n$-th pre-renormalization of $\left.f_{\theta}\right|_{\partial \Delta}$ as the first return map of the union of the arcs $A_{n}=\left[c, f_{\theta}^{q_{n}}(c)\right]$, $A_{n+1}=\left[f_{\theta}^{q_{n}}(c), c\right]$, which is

$$
\left(\left.f^{q_{n+1}}\right|_{A_{n}},\left.f^{q_{n}}\right|_{A_{n+1}}\right) .
$$

McMullen [McM3] defines renormalizations of Siegel quadratics using complexified versions of (2.5) similar to de Faria's holomorphic commuting pairs (cf. [dF1, dF2 ): 
Definition 2.4 (McMullen's holomoprhic pairs). Let $U_{1}, U_{2}, V$ be quasidisks in $\mathbb{C}$ with $U_{i} \subset V$. A holomorphic pair consists of two homeomorphisms $g_{i}: \overline{U_{i}} \mapsto \bar{V}$ univalent on the interior, such that:

- $V \backslash \overline{U_{1} \cup U_{2}}$ is also a quasidisk;

- $\overline{U_{i}} \cap \partial V=I_{i}$ is an arc;

- $g_{i}\left(I_{i}\right) \subset I_{1} \cup I_{2}$ for $i=1,2$;

- $\overline{U_{1}} \cap \overline{U_{2}}=\{c\}$ is a single point.

The filled Julia set of a holomorphic pair $\left(g_{1}, g_{2}\right)$ is by definition the set $K\left(g_{1}, g_{2}\right)$ of nonescaping points for this dynamical system.
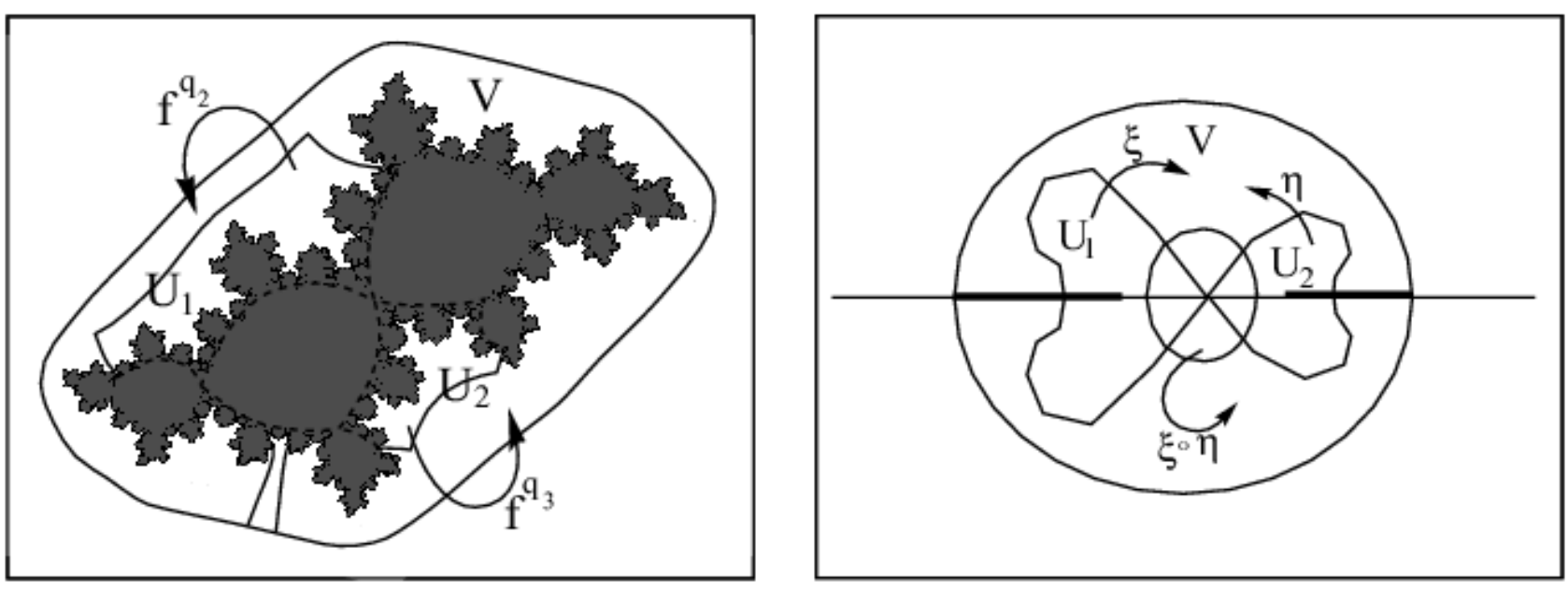

Figure 2. On the left, the second pre-renormalization of the golden mean Siegel disk; on the right a de Faria's holomorphic commuting pair.

A pre-renormalization of $f_{\theta}$ in the sense of McM3 is a holomorphic pair

$$
p \mathcal{R}_{\mathrm{McM}}^{n} f=\left(f^{q_{n}}: U_{1}^{n} \rightarrow V^{n}, f^{q_{n+1}}: U_{2}^{n} \rightarrow V^{n}\right)
$$

such that $I_{1}^{n}=A_{n}$ and $I_{2}^{n}=A_{n+1}$. The corresponding renormalization $\mathcal{R}_{\mathrm{McM}}^{n} f$ is the above holomorphic pair linearly rescaled by a mapping sending $c$ to 0 and the other endpoint of $A_{n}$ to 1 .

Proposition 2.6 ([McM3]). A pre-renormalization (2.6) exists for every value of $n \in \mathbb{N}$.

This evidently follows from Theorem 2.2 and the surgery construction of Theorem 2.5. Below we briefly outline a different argument due to McMullen, making use of the local connectivity of the Julia set $J\left(f_{\theta}\right)$.

The domain $V^{n}$ is taken to be the annulus between the boundary of $\Delta$ and some equipotential $E$ of the Julia set of $f_{\theta}$, from which a slight thickening of the external ray landing 
at $f_{\theta}(c)$ is removed. The domain $\overline{U_{1}^{n}}$ is then the univalent pull-backs of $\overline{V^{n}}$ along the orbit

$$
I_{i} \mapsto f\left(I_{1}^{n}\right) \mapsto \cdots \mapsto f^{q_{n}}\left(I_{1}^{n}\right),
$$

and similarly for $U_{2}^{n}$.

A holomorphic pair $\mathcal{P}$ (2.6) under the the inverse of the surgery map $\psi(2.4)$ and after Schwarz reflection gives rise to a holomorphic commuting pair $\mathcal{H}=\mathcal{R}^{k} F_{\theta}$. Let us say that $\mathcal{P}$ has a complex bound $\mu$ if $\mathcal{H}$ possesses this bound.

Let us now fix $N \in \mathbb{N}$ and set

$$
\theta_{*}=\frac{1}{N+\frac{1}{N+\frac{1}{\cdots}}}
$$

Denote $p_{n} / q_{n}$ the $n$-th convergent of this infinite continued fraction.

Proposition 2.7 ([McM3]). There exists a constant $K>1$ such that the following holds. Every two pre-renormalizations (2.6) of $f_{\theta_{*}}$ constructed as above are $K$-quasiconformally conjugate. The conjugacy extends (anti) conformally to a neighborhood of $c$ in $\Delta$.

Theorem 2.8 ([McM3] ). Suppose $g$ is any analytic mapping with a golden-mean Siegel disk whose boundary is a quasicircle containing a single simple critical point, and let $\left(g_{1}, g_{2}\right)$ be a pre-renormalization of $g$ in the above sense. Assume that for some $n \in \mathbb{N}$ the holomorphic pair $\left(g_{1}, g_{2}\right)$ is quasiconformally conjugate to the $n$-th pre-renormalization of $f_{\theta_{*}}$ and the conjugacy extends (anti) conformally to a neighborhood of $c$ in $\Delta$. Then the extended conjugacy $\psi$ is $C^{1+\alpha}$-(anti) conformal at the critical point $c$.

The proof is based on the following concept developed in [McM2]:

Definition 2.5. A point $x$ is a measurable deep point of a compact $\Lambda \subset \mathbb{C}$ if there exists $\delta>0$ such that for all $r>0$

$$
\operatorname{area}\left(D_{r}(x) \backslash \Lambda\right)=O\left(r^{2+\delta}\right)
$$

The following theorem about measurable deep points appeared in [McM2]:

Theorem 2.9 (Boundary conformality). Let $\phi: \mathbb{C} \rightarrow \mathbb{C}$ be a quasiconformal map with $\bar{\partial} \phi=0$ on a measurable set $\Omega$, and let $x$ be a measurable deep point of $\Omega$. Then $\phi$ is $C^{1+\alpha}$-conformal at $x$.

For the holomorphic pair

$$
g_{n}=\left(g_{n}^{1}, g_{n}^{2}\right): \cup U_{n}^{i} \rightarrow U_{n}
$$

which is the $n$-th pre-renormalization of $f_{\theta_{*}}$ in the sense of McMullen the filled Julia set $K\left(g_{n}^{1}, g_{n}^{2}\right)$ is a subset of $J\left(f_{\theta}\right)$ and hence has zero area, and no measurable deep points. McMullen considers its thickening

$$
K_{\epsilon}\left(g_{n}^{1}, g_{n}^{2}\right)=K\left(g_{n}^{1}, g_{n}^{2}\right) \cup_{\ell>0}\left(g_{n}\right)^{-\ell}\left(D_{\epsilon}(c) \backslash U_{n}\right),
$$


and shows that for every $\epsilon>0$ the critical point $c$ is a measurable deep point of this set. The conjugacy constructed in Proposition 2.7 is then $C^{1+\alpha}$-conformal by Theorem 2.9. Denote $\mathcal{R}_{\mathrm{McM}}^{n} f_{\theta_{*}}$ the $n$-th pre-renormalization $g_{n}$ rescaled linearly so that $I_{1}^{n}$ is bounded by 0 and 1 , with $c$ mapping to 0 . Applying Theorem 2.8 to the particular case of these renormalized pairs, we have

Corollary 2.10. The renormalizations $\mathcal{R}_{M c M}^{n} f_{\theta_{*}}$ converge geometrically fast in $n$ with respect to the uniform metric on compact sets.

Denoting the limiting pair $(\hat{\omega}, \hat{v})$ we see that it is fixed under the operation $\mathcal{R}_{\mathrm{McM}}$. Moreover, if $g$ is as in Theorem 2.8 then

$$
\mathcal{R}_{\mathrm{McM}}^{n} g \rightarrow(\hat{\omega}, \hat{v})
$$

again at a geometric rate.

Cylinder renormalization. The cylinder renormalization was introduced in [Ya3], for a detailed discussion we refer the reader to that paper. Firstly, let us define some function spaces. Denote $\pi$ the natural projection $\mathbb{C} \rightarrow \mathbb{C} / \mathbb{Z}$, and $p: \mathbb{C} / \mathbb{Z} \rightarrow \overline{\mathbb{C}}$ the conformal isomorphism given by $p(z)=e^{2 \pi i z}$. For a topological disk $W \subset \mathbb{C}$ containing 0 and 1 we will denote $\mathbf{A}_{W}$ the Banach space of bounded analytic functions in $W$ equipped with the sup norm. Let us denote $\mathbf{C}_{W}$ the Banach subspace of $\mathbf{A}_{W}$ consisting of analytic mappings $h: W \rightarrow \mathbb{C}$ such that $h(0)=0$ and $h^{\prime}(1)=0$.

The cylinder renormalization operator is defined as follows. Let $f \in \mathbf{C}_{W}$. Suppose that for $n \in \mathbb{N}$ there exists a simple arc $l$ which connects a fixed point $a$ of $f^{n}$ to 0 , and has the property that $f^{n}(l)$ is again a simple arc whose only intersection with $l$ is at the two endpoints. Let $C_{f}$ be the topological disk in $\mathbb{C} \backslash\{0\}$ bounded by $l$ and $f^{n}(l)$. We say that $C_{f}$ is a fundamental crescent if the iterate $\left.f^{-n}\right|_{C_{f}}$ mapping $f^{n}(l)$ to $l$ is defined and univalent, and the quotient of $\overline{C_{f} \cup f^{-n}\left(C_{f}\right)} \backslash\{0, a\}$ by the iterate $f^{n}$ is conformally isomorphic to $\mathbb{C} / \mathbb{Z}$. Let us denote $R_{f}$ the first return map of $C_{f}$, and let us assume that this map has a critical point $z$ corresponding to the orbit of 0 . Let $g$ be the map $R_{f}$ becomes under the above isomorphism, mapping $z$ to 0 , and $h=p^{-1} \circ g \circ p$. We say that $f$ is cylinder renormalizable, if $h \in \mathbf{C}_{V}$ for some $V$, and call $h$ a cylinder renormalization of $f$.

Proposition 2.11. Suppose $f \in \mathbf{C}_{W}$ is cylinder renormalizable, and its renormalization $h_{f}$ is contained in $\mathbf{C}_{V}$. Denote $C_{f}$ the fundamental crescent corresponding to the renormalization. Then the following holds.

- Every other fundamental crescent $C_{f}^{\prime}$ with the same endpoints as $C_{f}$, and such that $C_{f}^{\prime} \cup C_{f}$ is a topological disk, produces the same renormalized map $h_{f}$.

- There exists an open neighborhood $U(f) \subset \mathbf{C}_{W}$ such that every map $g \in \mathbf{C}_{W}$ is cylinder renormalizable, with a fundamental crescent $C_{g}$ which can be chosen to move continuously with $g$.

- Moreover, the dependence $g \mapsto h_{g}$ of the cylinder renormalization on the map $g$ is an analytic mapping $\mathbf{C}_{W} \rightarrow \mathbf{C}_{V}$. 
Proof. The arguments from [Ya3] apply mutatis mutandis.

Let us now concentrate on the case of Siegel quadratics with a golden rotation number.

Theorem 2.12. Let $f_{\theta_{*}}$ be as above. There exists a sequence $g_{n}, n \in \mathbb{N}$ of cylinder renormalizations of $f_{\theta_{*}}$ with the following properties.

(I) There exists an increasing sequence of natural numbers $k_{n}$ such that $g_{n}$ is a cylinder renormalization of $f_{\theta_{*}}$ with period $q_{k_{n}}$. For every $n$, the map $g_{n}$ has a Siegel disk with rotation number $\theta_{*}$ centered at the origin, whose boundary is a quasicircle, containing the critical point 1.

(II) Let

$$
P_{n}: U_{1}^{n} \cup U_{2}^{n} \rightarrow V^{n}
$$

be a sequence of holomorphic pairs (2.6) with a uniform complex bound $\mu$. There exists $K \in \mathbb{N}$ such that for every $n$ the fundamental crescent $C_{n}$ corresponding to $g_{n}$ is contained in the union of the closures of the domains $U_{1}^{n-K}$ and $\Delta$.

(III) Finally, for $n_{2}>n_{1}$, the map $g_{n_{2}}$ is a cylinder renormalization of $g_{n_{1}}$.

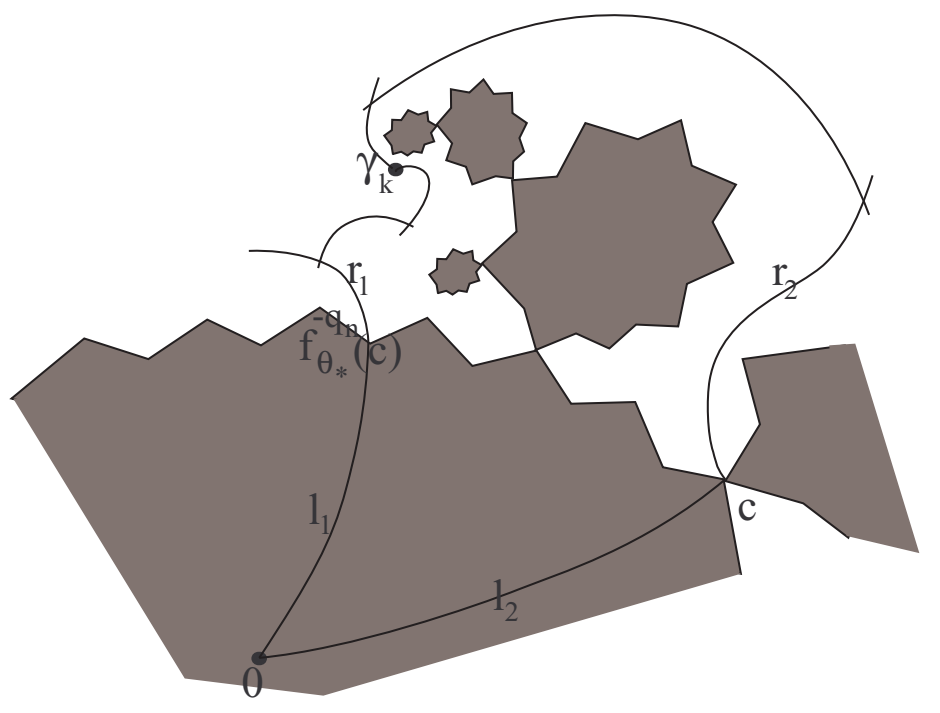

FiguRE 3.

We will give two different proofs of the above theorem:

Proof of Theorem 2.19 using the surgery on a Blaschke product. Let $F \equiv F_{\theta_{*}}$ be the Blaschke product (2.2). Denote $\zeta_{n}$ the $n$-th renormalization $\mathcal{R}^{n} F$ of this critical circle mapping. Each $\zeta_{n}$ is a commuting pair in the Epstein class, and therefore is cylinder renormalizable (Lemma 7.6 of [Ya3]). By complex a priori bounds (Theorem 2.2) we can choose a fundamental crescent $W_{n}$ for $\zeta_{n}$ so that $W_{n+M} \subset W_{n}$ for some fixed $M \in \mathbb{N}$. We may, moreover, 
select $W_{n}$ compactly contained in the domain of the holomorphic commuting pair $\mathcal{H}_{n}$ of Theorem 2.2. Denote $W_{n}^{+}=W_{n} \cap \mathbb{H}$ the upper half of the fundamental crescent. Let $p$ and $F^{q_{n}}(p)$ be the points of intersection of the boundary of $W_{n}$ with the unit circle. Let $\phi: \mathbb{D} \rightarrow \mathbb{D}$ be the quasiconformal mapping of $(2.3)$ and $\psi$ be the change of coordinates (2.4). Let $a=\phi(p)$ so that $b=r_{\theta_{*}}^{q_{n}}(a)=\phi\left(F^{q_{n}}(p)\right)$, and denote $L \subset \mathbb{D}$ the domain bounded by the line segments $[0, a],[0, b]$ and the arc $[a, b] \subset S^{1}$. Set $H_{n}=W_{n}^{+} \cup \phi^{-1}(L)$ and $C_{n}=\psi^{-1}\left(H_{n}\right)$. By construction, $C_{n}$ is a fundamental crescent for $f_{\theta_{*}}$. Denote $h_{n}$ the corresponding cylinder renormalization. Setting $g_{n}=h_{M n}$ we have the required properties.

Proof of Theorem 2.12 using an idea of X. Buff (private communication). We will explicitly construct a sequence of fundamental crescents $C_{k}$ for the map $f_{\theta_{*}}$. Denote $l_{1}, l_{2} \subset \bar{\Delta}$ the internal rays of the Siegel disk terminating at the endpoints of $\left[c, f_{\theta_{*}}^{-q_{n}}(c)\right] \subset \partial \Delta$, and let $r_{1}, r_{2}$ be segments of the two external rays of $K\left(f_{\theta_{*}}\right)$ landing at the same two points. Let $\gamma_{k}$ be the repelling fixed point of $f_{\theta_{*}}^{q_{k}}$ in $U_{1}^{k}$. Let $Q_{k} \ni \gamma_{k}$ be a linearizing neighborhood of $\gamma_{k}$, and $w_{k}: Q_{k} \rightarrow \mathbb{D}$ be the linearizing coordinate, conjugating $f_{\theta_{*}}^{q_{k}}$ to $z \mapsto \lambda_{k} z$ where $\lambda_{k}=\left(f_{\theta_{*}}^{q_{k}}\right)^{\prime}\left(\gamma_{k}\right)$. Denote

$$
\Psi:\{\operatorname{Re}(z)<0\} \rightarrow \mathbb{D}
$$

the exponential map $z \mapsto e^{z}$, and let $L$ be the ray

$$
\{\operatorname{Re}(z)=\operatorname{Im}(z), \operatorname{Re}(z)<0\} .
$$

Denote $s_{2}$ an arc of the logarythmic spiral $w_{k}^{-1} \circ \Psi(L)$ which terminates at $\gamma_{k}$ and whose other endpoint lies outside of the filled Julia set $K\left(f_{\theta_{*}}\right)$. Set $s_{1}$ to be the component of the preimage $f_{\theta_{*}}^{-q_{k}}\left(s_{2}\right)$ which contains $\gamma_{k}$. Let $t_{2}$ be a curve consisting of an arc of an external ray and of an equipotential connecting $s_{2}$ with $r_{2}$, and $t_{1}$ its $f_{\theta_{*}}^{-q_{k}}$-preimage connecting $s_{1}$ with $r_{1}$. By construction, the curves $L_{i}=l_{i} \cup r_{i} \cup t_{i} \cup s_{i}$ are disjoint and $f_{\theta_{*}}^{q_{k}}\left(L_{1}\right)=L_{2}$. The local pictures of dynamics at 0 and at $\gamma_{k}$ imply that $L_{1}$ and $L_{2}$ bound a fundamental crescent $C_{k}$ of $f_{\theta_{*}}$ (cf. Figure 3). The map $h_{k}$ which is the corresponding cylinder renormalization satisfies the property (I). By Corollary 2.10 there exists $M \in \mathbb{N}$ such that for every $k$ the crescent $C_{M+k} \subset C_{k}$. Setting $g_{k}=h_{M k}$ we have the desired properties.

2.1. Parabolic renormalization. For further reference, we give here a brief definition of the relevant version of parabolic renormalization. We refer the reader to [Shi1, and also to $[\mathrm{EY}$. The latter reference contains a detailed discussion of parabolic renormalization for critical circle mappings.

Let $f \in \mathbf{C}_{U}$ have a fixed point $p$ with eigenvalue 1 . Assume further, that $p$ is a simple parabolic point, and denote $C^{A}$ and $C^{R}$ the attracting and repelling Fatou cylinders of $p$. The overlap of the attracting and repelling petals of $f$ induces an analytic Écalle-Voronin mapping $\mathcal{E}_{f}$ from open neighborhoods $W^{+}, W^{-}$of the ends $\oplus$, $\ominus$ of $C^{R}$ to $C^{A}$. We normalize the situation by requiring that $\left(\mathcal{E}_{f}\right)^{\prime}(\oplus)=1$. 
An arbitrary choice of an affine isomorphism $\tau: C^{A} \rightarrow C^{R}$ induces a dynamical system

$$
g_{\tau} \equiv \tau \circ \mathcal{E}_{f}: W^{+} \cup W^{-} \rightarrow C^{R} .
$$

Denote $e(z)=e^{2 \pi i z}$ the conformal isomorphism $C^{A} \rightarrow \mathbb{C} \backslash\{0\}$, sending the end $\oplus$ to the puncure at the origin. Now let $\left[a_{1}, a_{2}, \ldots, a_{n}, \ldots\right]$ be any formal continued fraction with $a_{i} \in \mathbb{N} \cup\{\infty\}$. If there is no symbol $\infty$ present in the sequence $\left[a_{i}\right]$, then the continued fraction converges to a well-defined $\theta \in \mathbb{R} \backslash \mathbb{Q}$. Otherwise, assuming that $i$ is the first position in which $\infty$ is encountered, we let $\theta$ to be the rational $\left[a_{1}, \ldots, a_{i-1}\right]$.

We set

$$
\mathcal{P}_{\theta} f=e \circ g_{\tau(\theta)} \circ e^{-1}
$$

for the unique choice of $\tau(\theta)$ such that $\left(\mathcal{P}_{\theta} f\right)^{\prime}(0)=e^{2 \pi i \theta}$, and call it the parabolic renormalization of $f$ corresponding to $\theta$.

Parabolic renormalization can be seen as the limiting case of cylinder renormalization as seen from the following (cf. [Shi1, [EY]):

Theorem 2.13. For $f$ as above, there exists a neighborhood $\mathcal{U}(f) \subset \mathbf{C}_{U}$ such that the following holds. Every $h \in \mathcal{U}(f)$ with $h^{\prime}(0)=e^{2 \pi i t}, t \neq 0$ is cylinder renormalizable with period 1 .

Moreover, fix $\theta=\left[a_{1}, a_{2}, \ldots, a_{n}, \ldots\right]$ with $a_{i} \in \mathbb{N} \cup\{\infty\}$. Let $h_{i} \in \mathcal{U}(f)$ converge to $f$, and have $\left(h_{i}\right)^{\prime}(0)=e^{2 \pi i t_{i}}$. When $t_{i} \neq 0$, let $g_{i}$ be the cylinder renormalization of $h_{i}$ of period 1 . When $t_{i}=0$, set $g_{i}=\mathcal{P}_{\theta} h_{i}$.

Assume that $\left(g_{i}\right)^{\prime}(0) \rightarrow e^{2 \pi i \theta}$. Then

$$
g_{i} \rightarrow \mathcal{P}_{\theta} f
$$

uniformly in some neighborhood of 0.

\section{Proof of the MAIN Theorem}

\section{The definition of $\mathcal{R}_{\mathrm{c} y l}$ and the existence of a fixed point $\hat{f}$.}

Proposition 3.1. Let $g_{n}$ be the sequence of cylinder renormalizations of $f_{\theta_{*}}$ constructed in Theorem 2.12. There exists $M \in \mathbb{N}$ and a domain $U \supset\{0,1\}$ such that for $n \geq M$ the renormalizations $g_{n} \in \mathbf{C}_{U}$, and converge geometrically fast in the uniform topology to a map $\hat{f} \in \mathbf{C}_{U}$. The map $\hat{f}$ has a Siegel disk $\Delta_{\hat{f}}$ which is compactly contained in $U$.

Proof. Let $K$ be as in Theorem 2.12. By Theorem 2.8 $\mathcal{R}_{\mathrm{McM}}^{K n} f$ converge geometrically fast to a holomorphic pair $(\hat{\omega}, \hat{v})$ which is fixed under $\mathcal{R}_{\mathrm{McM}}$. This and Theorem 2.12 imply the desired claim.

Proposition 3.2. There exists a topological disk $W \ni \Delta_{\hat{f}}$ such that the following holds. For every topological disk $V$ with $W \ni V \ni \Delta_{\hat{f}}$ we have:

(I) Denote $g$ the restriction $\left.\hat{f}\right|_{V}$. Then the map $g$ has a cylinder renormalization $\tilde{g}$ whose domain of definition compactly contains $W$ and such that $\left.\left.\tilde{g}\right|_{W} \equiv \hat{f}\right|_{W}$. 
(II) Moreover, there exists $n$ such that the $n$-th pre-renormalization in the sense of McMullen (2.6)

$$
p \mathcal{R}_{M c M}^{n}: U_{1}^{n} \cup U_{2}^{n} \rightarrow V^{n}
$$

exists for $g$.

(III) Finally, let $J_{i}$ be an element of the $n$-th dynamical partition (2.1) of $\left.g\right|_{\partial \Delta_{\hat{f}}}$ corresponding to the orbit of 0 , and $g^{k}\left(J_{i}\right) \subset \overline{U_{1}^{n} \cup U_{2}^{n}}$. Then the inverse branch $\tau_{i}$ mapping $g^{k}\left(J_{i}\right)$ to $J_{i}$ univalently extends to $\left(V^{n} \backslash \partial \Delta_{\hat{f}}\right) \cup g^{k}\left(J_{i}\right)$ and maps it to a subset of $V$.

Proof. We will make use of the quasiconformal conjugacy $\psi=\psi_{\theta_{*}}$ (2.4) conjugating the quadratic map $f_{\theta_{*}}$ to the modified Blaschke product $\tilde{F}_{\theta_{*}}$. Let $V$ be any domain compactly containing $\Delta_{\hat{f}}$. Set $X=\psi(V) \backslash \overline{\mathbb{D}}$, and $Y$ to be the union of $X$ with its reflection in $S^{1}$, together with the $\operatorname{arc} \psi(V) \cap S^{1}$. Let $G=\left.F_{\theta_{*}}\right|_{Y}$. By complex a priori bounds (Theorem 2.2) there exists $N$ such that for every analytic critical circle map $h$ in $Y$ with a single critical point, for $n \geq N$ the commuting pair $p \mathcal{R}^{n} G$ extends to a holomorphic commuting pair $\mathcal{H}_{h}^{n} \in \mathbf{H}(\mu)$. By a compactness argument, $N=N(Y)$.

The pull-back of the "upper half" of the holomorphic commuting pair $\mathcal{H}_{G}^{n}$ by the conjugacy $\psi$ is a holomorphic pair

$$
p \mathcal{R}_{\mathrm{McM}}^{n}: U_{1}^{n} \cup U_{2}^{n} \rightarrow V^{n},
$$

which implies part (II). Note that the universality of the bound $\mu$ and Theorem 2.12 (II) imply that there exists $W$ independent of the initial choice of $V$ such that (I) holds. Part (III) follows from the same considerations.

Definition 3.1. Let us select a subdomain $V \Subset W$, and $n \in \mathbb{N}$ as in the previous Proposition. Let $U \Subset W$ be an open neighborhood of $\Delta_{\hat{f}}$ such that

$$
U \Subset \cup \tau_{i}\left(V^{n}\right) \cup \overline{\Delta_{\hat{f}}} \text {, and } U \ni \cup \tau_{i}\left(U_{i}^{n}\right)
$$

By Proposition 3.2 (I) there exists a cylinder renormalization transforming $\left.\hat{f}\right|_{U}$ to $\left.\hat{f}\right|_{W}$. By Proposition 2.11 it extends to an analytic operator from an open subset of $\mathbf{C}_{U}$ to $\mathbf{C}_{U}$. We will denote this operator $\mathcal{R}_{\mathrm{cyl}}$, and call it the cylinder renormalization operator.

The expanding direction of $\mathcal{R}_{\text {cyl }}$. Let us denote $\mathcal{L}=D_{\hat{f}} \mathcal{R}_{\mathrm{cyl}}: \mathbf{C}_{U} \rightarrow \mathbf{C}_{U}$. We first establish that:

Proposition 3.3. The operator $\mathcal{L}$ is compact.

Proof. Denote $B_{1}$ the unit ball in $\mathbf{C}_{U}$ and let $v \in B_{1}$. By definition of $\mathcal{R}_{\text {cyl }}$, the vector field $\mathcal{L} v$ is an analytic vector field in the domain $W \ni U$. Denote $C \Subset U$ a fundamental crescent which corresponds to the cylinder renormalization operator, and let $\Phi: C \rightarrow \hat{\mathbb{C}}$ be its uniformization. The first return map $R_{C}$ of $C$ under $\left.\hat{f}\right|_{U}$ is a bounded piecewise analytic map. The restriction of $R_{C}$ to $\Phi^{-1}(U)$ is a finite collection of iterates $\hat{f}^{j}$, and the 
compactness considerations imply that there exists $C$ independent of $v$ such that $\left\|\left.\mathcal{L} v\right|_{U}\right\|<$ $C$. Since the $C$-bounded functions in $\mathbf{C}_{U}$ which analytically extend to $W$ form a compact set, the image $\overline{\mathcal{L}\left(B_{1}\right)}$ is compact.

Proposition 3.4. The operator $\mathcal{L}$ has an eigenvalue $\lambda$ with $|\lambda|>1$.

Proof. Let $v(z)$ be a vector field in $\mathbf{C}_{U}$,

$$
v(z)=v^{\prime}(0) z+o(z)
$$

Denote $\gamma_{v}$ the quantity

$$
\gamma_{v}=\frac{v^{\prime}(0)}{\hat{f}^{\prime}(0)}=e^{-2 \pi i \theta_{*}} v^{\prime}(0)
$$

For a smooth family

$$
\hat{f}_{t}(z)=\hat{f}(z)+t v(z)+o(t)
$$

we have

$$
\hat{f}_{t}(z)=\alpha_{t}^{v}(z)\left(\hat{f}^{\prime}(0) z+o(z)\right), \text { where } \alpha_{t}(0)=1+t \gamma_{v}+o(t) .
$$

The $q_{m+1}$-st iterate

$$
\hat{f}_{t}^{q_{m+1}}(z)=\left(\alpha_{t}^{v}(z)\right)^{q_{m+1}}\left(\left(\hat{f}^{\prime}(0)\right)^{q_{m+1}} z+o(z)\right) .
$$

In the neighborhood of 0 the renormalized vector field $\mathcal{L} v$ is obtained by applying a uniformizing coordinate

$$
\Psi(z)=(z+o(z))^{\beta}, \text { where } \beta=\frac{1}{\theta_{*} q_{m} \bmod 1} .
$$

Hence,

$$
\alpha_{t}^{\mathcal{L} v}(0)=\left[\left(\alpha_{t}^{v}(0)\right)^{q_{m+1}}\right]^{\beta}
$$

so

$$
\gamma_{\mathcal{L} v}=\Lambda \gamma_{v}, \text { where } \Lambda=\beta q_{m+1}>1
$$

Hence the spectral radius

$$
R_{\mathrm{Sp}}(\mathcal{L} v)>1
$$

and since every non-zero element of the spectrum of a compact operator is an eigenvalue, the claim follows.

Stable direction. Denote $\mathcal{W} \subset \mathbf{C}_{U}$ the collection of mappings $f$ such that $f^{\prime}(0)=e^{2 \pi i \theta_{*}}$. Inou and Shishikura's results. Inou and Shishikura Shi2 have recently established the following result:

Theorem 3.5. There exist $N_{0} \in \mathbb{N}$, a pair of topological disks $\widetilde{W} \ni W \ni 0$, and an open neighborhood $\mathcal{V} \subset \mathbf{C}_{W}$ so that the following is true. 
- Let $\theta=\left[a_{1}, a_{2}, \ldots\right]$ with $a_{i} \in \mathbb{N} \cup \infty$ and $a_{i} \geq N_{0}$. For every $f \in \mathcal{V}$ with $f^{\prime}(0)=e^{2 \pi i \theta}$ we have the following. If $a_{1} \neq \infty$, then $f$ is cylinder renormalizable with period 1 , and the corresponding cylinder renormalization $g \in \mathcal{V}$. Otherwise,

$$
\mathcal{P}_{\left[a_{2}, a_{3}, \ldots\right]} f \in \mathcal{V} \cap \mathbf{C}_{\widetilde{W}} .
$$

- Moreover, consider the quadratic polynomial $f=f_{\theta}(z)$. Set $g_{i}$ to be the sequence of cylinder/parabolic renormalizations of $f$ with

$$
\left(g_{i}\right)^{\prime}(0)=e^{2 \pi i\left[a_{i+1}, a_{i+2}, \ldots\right]} .
$$

Then there exists $j \in \mathbb{N}$ such that $g_{j} \in \mathcal{V}$.

- Finally, the neighborhood $\mathcal{V}$ can be chosen sufficiently small, so that, in particular, for every $f \in \mathcal{V}$ the critical point 1 is not fixed.

Note that the claim (V) of the Main Theorem immediately follows from the above result.

Proposition 3.6. There exists an open neighborhood $\mathcal{U} \subset \mathbf{C}_{U}$ of $\hat{f}$ such that there exists $N \in \mathbb{N}$ such that for every $f \in \mathcal{W} \cap \mathcal{U}$ the following holds:

(I) the boundary of the Siegel disk $\partial \Delta_{f}$ is a quasicircle containing the critical point 1;

(II) the pair of mappings

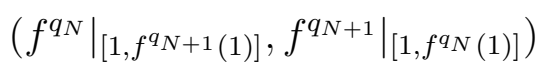

extends to a holomorphic pair, which is quasiconformally conjugate to $(\hat{\omega}, \hat{v})$ (2.7) in such a way, that the conjugacy conformally extends to the Siegel disks.

Proof. From Theorem 3.5 there exists a neighborhood $\mathcal{U}$ such that for every $f \in \mathcal{U}$ the critical orbit $\left\{f^{n}(1)\right\}$ is infinite and compactly contained in $\mathcal{U}$. Since the dependence $f \mapsto f^{n}(1)$ is holomorphic, the Bers-Royden Theorem implies that the closure $\overline{\left\{f^{n}(1)\right\}}$ is a $K$-quasicircle $\Upsilon_{f}$ for some $K=K(\mathcal{U})>1$. Given the invariance of $\Upsilon_{f}$, and considerations of the Denjoy-Wolff Theorem, we see that $\Upsilon_{f}=\partial \Delta_{f}$. The standard pull-back argument implies that $f$ is $K_{1}=K_{1}(\mathcal{U})$-quasiconformally conjugate to $\hat{f}$ on a subdomain $V \subset U$ with $\Delta_{f} \Subset V$.

By Proposition 3.2 the neighborhood $\mathcal{U}$ may be chosen small enough so that (II) holds for some $N \in \mathbb{N}$.

Proposition 3.7. There exists a codimension one subspace $T$ of $\mathbf{C}_{U}$ such that

$$
R_{s p}\left(\left.\mathcal{L}\right|_{T}\right)<1
$$

Proof. We take $T$ to be the tangent space to the codimension one submanifold $\mathcal{U} \cap \mathcal{W} \subset \mathbf{C}_{U}$ at the point $\hat{f}$. By McMullen's Theorem 2.8, for every map $f \in \mathcal{U}$,

$$
\mathcal{R}_{\text {cyl }}^{k} f \rightarrow \hat{f}
$$

geometrically fast. The Spectral Theorem for compact operators implies that

$$
R_{\mathrm{sp}}\left(\left.\mathcal{L}\right|_{T}\right)<1
$$




\section{Appendix A. Application to the Measure Problem}

The question of existence of polynomial Julia sets with positive area has long been a central problem in Holomorphic Dynamics. Some years ago Douady has outlined a program for constructing such sets in the family of quadratic polynomials. Chéritat Ch in his thesis made a major progress in the program, reducing it to several renormalizationrelated conjectures. Very recently, Buff and Chéritat $\mathrm{BC}$ have announced the construction of some positive measure examples. In their approach, they used the work of Inou \& Shishikura Shi2] to resolve the conjectures of Chéritat.

In this Appendix, we demonstrate how the same result follows from hyperbolicity of Siegel renormalization (Theorem 1.1). Given that part (VI) of Theorem 1.1 also relies on the work of Inou and Shishikura, this approach is not much different from that taken by Buff and Chéritat. The use of the renormalization theorem, however, makes for a shorter proof, and clarifies the connection between hyperbolicity of $\mathcal{R}_{\text {cyl }}$ and positive measure. The latter could be particularly useful in the further study of measure of Cremer and Siegel Julia sets by renormalization techniques.

We formulate a theorem which by [Ch] implies that some quadratic Julia sets have positive measure:

Theorem A.1. Let $\left[a_{1}, \ldots, a_{k}\right]$ be any finite continued fraction with $a_{i} \geq N_{0}$ from Theorem [3.5. Fix $N \geq N_{0}$. Denote $\theta_{j}$ the finite continued fraction

$$
\theta_{j}=[a_{1}, \ldots, a_{k}, \underbrace{N, N, \ldots, N}_{j}, \infty]
$$

Let $\mathbb{C} / \mathbb{Z} \simeq C^{R}$ be the repelling cylinder of the quadratic polynomial $f_{\theta_{j}}$. Denote $\hat{K}_{j} \subset C^{R}$ the projection of $K\left(f_{\theta_{j}}\right)$, and let $C_{j}$ be its complement $C^{R} \backslash \hat{K}_{j}$. Then the following holds:

(I) Denote

$$
h_{j}=\sup \left(\operatorname{Im} z_{1}-\operatorname{Im} z_{2}\right), \text { for } z_{1}, z_{2} \in C_{j} .
$$

Then $\sup h_{j}<\infty$.

$$
\operatorname{Area}\left(C_{j}\right) \rightarrow 0 \text {. }
$$

Note that (I) follows immediately from Theorem 3.5 and compactness considerations. We will proceed to deriving (II).

Jellouli and Chéritat have shown (see Ch]):

Theorem A.2. For every finite continued fraction $\left[a_{1}, \ldots, a_{k}\right]$ denote

$$
\theta_{j}=[a_{1}, \ldots, a_{k}, \underbrace{N, N, \ldots, N}_{j}, \infty] \text {, and } \theta_{\infty}=\lim _{j \rightarrow \infty} \theta_{j} .
$$

Then

$$
\text { Area } K\left(f_{\theta_{\infty}}\right) \backslash K\left(f_{\theta_{j}}\right) \longrightarrow 0 \text {. }
$$

We also recall the following fact proven in [Ya1]: 
Lemma A.3. Let $\theta_{\infty}$ be as above, and denote $\Delta=\Delta_{\theta_{\infty}}$ the Siegel disk of the quadratic polynomial $f_{\theta_{\infty}}$. Then

$$
\liminf _{\epsilon \rightarrow 0} \frac{\operatorname{meas} U_{\epsilon}(\Delta) \backslash K\left(f_{\theta_{\infty}}\right)}{\operatorname{meas} U_{\epsilon}(\Delta)}=0
$$

We remark that by later work of McMullen [McM3], in the above expression lim inf can be replaced with lim, and in fact, the convergence occurs at a geometric rate. However, this stronger result will not be needed.

The idea of the proof of Theorem A.1 is rather straightforward. As seen from the above lemma, the basin of the Siegel disk of $f_{\theta_{\infty}}$ occupies most of the area in a sequence of arbitrarily small disks around the critical point. Hence, for the renormalization fixed point $\hat{f}$, the Siegel basin has full measure in the cylinder. As implied by the hyperbolicity of renormalization, all sufficiently high cylinder renormalizations of $f_{\theta_{j}}$ are contained in a small neighbourhood of $\hat{f}$. From this we will conclude that the parabolic basin of $f_{\theta_{j}}$ on a sufficiently small scale contains most of the Siegel basin of $\hat{f}$ with thin cusps removed. By the result of Jellouli and Chéritat the total area of the removed cusps can be made arbitrary small by selecting large enough $j$. Hence, most of the measure in a fundamental crescent of $f_{\theta_{j}}$ will be occupied by the parabolic basin. Now we proceed to formalize this discussion.

Proof of Theorem A.1. First of all, observe that by Lemma A.3, and Theorem 1.1, for every $\epsilon>0$ and every disk $D \subset \mathbb{C} / \mathbb{Z}$ there exists $K_{0} \in \mathbb{N}$ such that for every $K>K_{0}$ there exists $M \in \mathbb{N}$ for which the following holds. Denote $g_{K}=\mathcal{R}_{\text {cyl }}^{K}\left(f_{\theta_{\infty}}\right)$, and let $B_{K}$ be the projection on the domain of $g_{K}$ of $\left(f_{\theta_{\infty}}\right)^{-M}(\Delta)$. Then

$$
\frac{\operatorname{Area}\left(D \backslash B_{K}\right)}{\operatorname{Area}(D)}<\epsilon .
$$

Now select a point $z \in C_{j}$ and let $\zeta$ be any lift of this point. Let us fix $\epsilon>0$ and $M \in \mathbb{N}$ and select a sufficiently large $j \in \mathbb{N}$ and $K+M<j$ such that the above inequality holds. Let $\left(\omega: U_{1} \rightarrow V, v: U_{2} \rightarrow V\right)$ be a McMullen renormalization of $g_{K}$.

Given that the point $\zeta$ is not in the filled Julia set of $f_{\theta_{j}}$, we necessarily have one of the following two scenarios:

(*) either there exists $n \in \mathbb{N}$ such that $\zeta_{n} \equiv\left(f_{\theta_{j}}\right)^{n}(\zeta)$ is contained in the domain $V \backslash\left(U_{1} \cup U_{2}\right)$

$(* *)$ or there exists $n$ such that $\zeta_{n} \in \Delta$.

In the case $(*)$, consider a disk $D_{n} \ni \zeta_{n}$ such that

$$
\frac{\operatorname{Area}\left(D_{n} \backslash f^{-M}(\Delta)\right)}{\operatorname{Area}\left(D_{n}\right)}<\epsilon \text {, and } \operatorname{dist}\left(D_{n}, \operatorname{Postcrit}\left(f_{\theta_{\infty}}\right)\right)>\operatorname{diam}\left(D_{n}\right) \text {. }
$$

Denote $D \ni \zeta$ its preimage, and $\tilde{D} \ni z$ the projection to $C^{R}$. By Koebe's Distortion Theorem and Theorem A.2 we have

$$
\operatorname{Area}\left(\tilde{D} \cap C_{j}\right) / \operatorname{Area}(\tilde{D})<\delta(\epsilon) \text { with } \delta(\epsilon) \underset{\epsilon \rightarrow 0}{\longrightarrow} 0,
$$


where Postcrit $\left(f_{\theta_{\infty}}\right)$ denotes the postcritical set.

In the case $(* *)$ we can select a disk $D_{n-1} \ni \zeta_{n-1}$ with the same properties, and the same considerations apply.

The statement of the theorem follows. 


\section{REFERENCES}

[BR] L. Bers \& H.L. Royden. Holomorphic families of injections. Acta Math. 157(1986), 259-286.

[BC] X. Buff, A. Chéritat. Ensembles de Julia quadratiques de mesure de Lebesgue strictement positive. Preprint, 2005.

[Ch] A. Chéritat. Recherche d'ensembles de Julia de mesure de Lebesgue positive. Thése, Orsay, 2001.

[dF1] E. de Faria. Proof of universality for critical circle mappings. Thesis, CUNY, 1992.

[dF2] E. de Faria. Asymptotic rigidity of scaling ratios for critical circle mappings. Ergodic Theory Dynam. Systems 19(1999), no. 4, 995-1035.

[dFdM1] E. de Faria and W. de Melo. Rigidity of critical circle mappings I. J. Eur. Math. Soc. (JEMS) $\mathbf{1}(1999)$, no. 4, 339-392.

[dFdM2] E. de Faria and W. de Melo. Rigidity of critical circle mappings II. J. Amer. Math. Soc., 13(2000), no. 2, 343-370.

[EY] A. Epstein \& M. Yampolsky. A universal parabolic map. Erg. Th. Dynam. Sys., to appear.

[FKS] M. Feigenbaum, L. Kadanoff, and S. Shenker. Quasi-periodicity in dissipative systems. A renormalization group analysis. Physica 5D (1982), 370-386.

[Her] M. Herman. Conjugaison quasi-symmetrique des homeomorphismes analytiques du cercle a des rotations. Manuscript.

[Lan1] O.E. Lanford. Renormalization group methods for critical circle mappings with general rotation number, VIIIth International Congress on Mathematical Physics (Marseille,1986), World Sci. Publishing, Singapore, 532-536, (1987).

[Lan2] O.E. Lanford. Renormalization group methods for critical circle mappings. Nonlinear evolution and chaotic phenomena, NATO adv. Sci. Inst. Ser. B:Phys.,176, Plenum, New York, 25-36, (1988).

[Lyu2] M. Lyubich. Renormalization ideas in conformal dynamics. Cambridge Seminar "Current Developments in Math.", May 1995. International Press, 1995. Cambridge, MA, 155-184.

[Lyu4] M. Lyubich. Feigenbaum-Coullet-Tresser Universality and Milnor's Hairiness Conjecture. Ann. of Math. (2) 149(1999), no. 2, 319-420.

[Lyu5] M. Lyubich. Almost every real quadratic map is either regular or stochastic. Annals of Math., to appear.

[MN] N.S. Manton, M. Nauenberg. Universal scaling behaviour for iterated maps in the complex plane. Commun. Math. Phys. 89(1983), 555-570.

[MP] R.S. MacKay, I.C. Persival. Universal small-scale structure near the boundary of Siegel disks of arbitrary rotation numer. Physica 26D(1987), 193-202.

[McM1] C. McMullen. Complex dynamics and renormalization. Annals of Math. Studies, v.135, Princeton Univ. Press, 1994.

[McM2] C. McMullen. Renormalization and 3-manifolds which fiber over the circle. Annals of Math. Studies, Princeton University Press, 1996.

[McM3] C. McMullen. Self-similarity of Siegel disks and Hausdorff dimension of Julia sets. Acta Math. 180 (1998), 247-292.

[Mes] B.D. Mestel. A computer assisted proof of universality for cubic critical maps of the circle with golden mean rotation number. PhD Thesis, University of Warwick, 1985.

[Mil] J. Milnor. Dynamics in one complex variable. Introductory lectures. Friedr. Vieweg \& Sohn, Braunschweig, 1999.

$[\mathrm{MvS}] \quad$ W. de Melo \& S. van Strien. One dimensional dynamics. Springer-Verlag, 1993.

[ORSS] S. Ostlund, D. Rand, J. Sethna, E. Siggia, Universal properties of the transition from quasiperiodicity to chaos in dissipative systems. Physica, $\mathbf{8 D}(1983), 303-342$.

[Pet] C. Petersen. Local connectivity of some Julia sets containing a circle with an irrational rotation, Acta Math., 177 (1996) 163-224. 
[Shi1] M. Shishikura. The Hausdorff dimension of the boundary of the Mandelbrot set and Julia sets. Ann. of Math. (2) 147(1998), no. 2, 225-267.

[Shi2] M. Shishikura. Renormalization for parabolic fixed points and their perturbations. J.H. Hubbard's 60's Birthday conference, Luminy, June 2005.

[Sieg] C.L. Siegel. Iteration of analytic functions. Ann. Math. 43(1942), 607-612.

[Stir] A. Stirnemann. Existence of the Siegel disc renormalization fixed point. Nonlinearity 7 (1994), no. 3, 959-974.

[Sul1] D.Sullivan. Quasiconformal homeomorphisms and dynamics, topology and geometry. Proc. ICM86, Berkeley, v. II, 1216-1228.

[Sul2] D.Sullivan. Bounds, quadratic differentials, and renormalization conjectures. AMS Centennial Publications. 2: Mathematics into Twenty-first Century (1992).

[Sw] G.Świastek. Rational rotation numbers for maps of the circle. Commun. Math. Phys. 119(1988), 109-128.

[Wi] M. Widom. Renormalisation group analysis of quasi-periodicity in analytic maps. Commun. Math. Phys. 92(1983), 121-136.

[Ya1] M. Yampolsky. Complex bounds for renormalization of critical circle maps, Erg. Th. ES Dyn. Systems. 19(1999), 227-257.

[Ya2] M. Yampolsky. The attractor of renormalization and rigidity of towers of critical circle maps, Commun. Math. Phys., 218(2001), no. 3, 537-568.

[Ya3] M. Yampolsky. Hyperbolicity of renormalization of critical circle Publ. Math. Inst. Hautes Etudes Sci. No. 96 (2002), 1-41.

[Ya4] M. Yampolsky. Renormalization horseshoe for critical circle maps. Commun. Math. Physics, 240(2003), 75-96.

[Yoc] J.C. Yoccoz. Il n'ya pas de contre-example de Denjoy analytique, C.R. Acad. Sci. Paris 298(1984) série I, 141-144. 